Obere Extremität $2011 \cdot 6: 77-77$ DOI 10.1007/s11678-011-0114-4 Online publiziert: 14. April 2011

(c) Springer-Verlag 2011

\author{
M. Scheibel \\ Centrum für Muskuloskeletale Chirurgie (CMSC), Campus-Mitte und \\ Campus-Virchow Klinikum, Charité-Universitätsmedizin Berlin
}

\title{
Akute und chronische Schultereckgelenkinstabilitäten
}

\section{Aktuelle Entwicklungen}

Akute und chronische Schultereckgelenkinstabilitäten stellen nach wie vor eine diagnostische und therapeutische Herausforderung für jeden schulterchirurgisch tätigen Unfallchirurgen bzw. Orthopäden dar. Gegenwärtig verfügbare Klassifikationssysteme erlauben nur eine unzureichende Kategorisierung zugrundeliegender kapsuloligamentärer bzw. muskulofaszialer Verletzungsmuster mit daraus resultierenden uni- bis mehrdirektionalen Instabilitäten. Während das Ausmaß der vertikalen Instabilität seit Langem in die therapeutische Entscheidungsfindung einfließt, wurde der horizontalen Instabilitätskomponente erst in den vergangenen Jahren vermehrt Aufmerksamkeit geschenkt. Verschiedene Arbeiten haben die posteriore Instabilität der Klavikula als einen möglichen negativen prognostischen Faktor herausgearbeitet. Neue nativ-radiologische Methoden im Rahmen der präoperativen Abklärung sollen in Zukunft eine bessere Einordnung der Pathologie gewährleisten und konkretere Therapieempfehlungen ermöglichen. In Anlehnung an diese Daten favorisieren aktuelle biomechanische Untersuchungen einen an das Instabilitätsmuster angepassten Therapieansatz. Neben der Wiederherstellung der vertikalen Stabilität bedarf es ggf. auch einer additiven akromioklavikulären Rekonstruktion, um eine relevante posteriore Instabilitätskomponente zu adressieren. Diese Empfehlungen spiegeln sich bereits in einigen modernen arthroskopisch-assistierten bzw. minimalinvasiven Techniken zur Behandlung akuter und chronischer Schultereckgelenkinstabilitäten wider. Während bei akuten Verletzungen weiterhin eine rein syntheti- sche Augmentation der rupturierten Bänder favorisiert wird, bedarf es in der chronischen Situation eines biologischen Ersatzes des korakoklavikulären und in einigen Fällen auch des akromioklavikulären Bandapparates. Auch hier geht der Trend hin zu anatomischen Rekonstruktionsverfahren unter Verwendung freier Sehnentransplantate, um eine Wiederherstellung der normalen Schultereckgelenkkinematik zu gewährleisten.

Ziel dieses Themenheftes ist es, neue Aspekte und Verfahren in der Diagnostik und Klassifikation von Schultereckgelenksprengungen aufzuzeigen und biomechanische Daten bzw. erste Behandlungsergebnisse von zukunftsorientierten arthroskopisch-assistierten bzw. minimalinvasiven Rekonstruktionstechniken vorzustellen.

In diesem Sinne wünsche ich Ihnen viel Spaß bei der Durchsicht des vorliegenden Themenheftes.

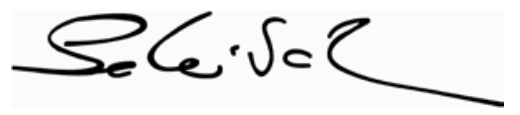

M. Scheibel

Korrespondenzadresse

PD Dr. M. Scheibel

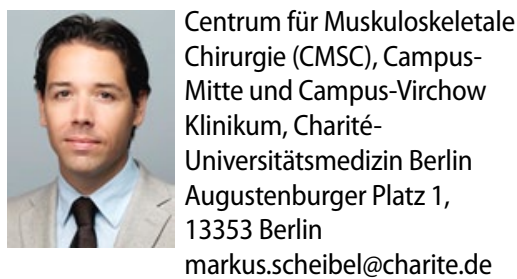

